\title{
Cannabis-Induced Psychosis in a Young Man With Traumatic Brain Injury
}

\author{
Albert Chang, MD ${ }^{1}$ - Bilen Kassu, MD' - Chinedu Ivonye, $\mathrm{MD}^{2}$ - Judith Volcy, DO³
}

A 21-year-old man was brought to the emergency department (ED) by his mother, who reported that the patient had been experiencing visual hallucinations and paranoia for the past 4 days. The mother denied a history of similar episodes and denied a family history of psychiatric disorders.

The patient had a medical history significant for traumatic brain injury (TBI) secondary to a motor vehicle collision that had occurred 5 years prior to presentation.

\section{Physical examination}

The patient had a guarded and irritated affect, saying, "I feel fine" when questioned. He reported having smoked marijuana and was orientated to person, place, and time. Despite this, the patient was physically agitated. Intravenous haloperidol, $5 \mathrm{mg}$, intravenous lorazepam, $1 \mathrm{mg}$, and oral diphenhydramine, $50 \mathrm{mg}$ were administered in the ED.

A urine toxicology screen was positive for cannabis. The patient's complete blood cell count, renal function and liver enzyme, thyroid-stimulating hormone, rapid plasma reagin, vitamin $\mathrm{B} 12$, and folate levels were within normal limits. An electrocardiogram was unremarkable. A magnetic resonance imaging (MRI) scan was performed, results of which showed advanced basal ganglia mineralization for the patient's age (Figure). A lumbar puncture was performed, and results were negative for infection. An electroencephalogram did not show any seizure activity.

Because new-onset schizophrenia was high on our differential diagnosis, a neurologist and psychiatrist were consulted. Because of the abnormalities on the MRI scan, bilateral striopallidodentate calcinosis was also considered in the differential diagnosis. However, it was determined that the lesions seen on the MRI were related to the patient's history of TBI.

Our final diagnosis was cannabisinduced psychosis in the setting of TBI. The patient's mentation returned to baseline by hospital day 4 after treatment with

\section{AFFILIATIONS:}

${ }^{1}$ Resident Physician, Department of Internal Medicine, Morehouse School of Medicine, Atlanta, Georgia ${ }^{2}$ Professor of Medicine, Department of Internal Medicine, Morehouse School of Medicine, Atlanta, Georgia

${ }^{3}$ Associate Professor of Medicine, Department of Internal Medicine, Morehouse School of Medicine, Atlanta, Georgia

\section{CITATION:}

Chang A, Kassu B, Ivonye C, Volcy J. Cannabis-induced psychosis in a young man with traumatic brain injury. Consultant. Published online January 13, 2022. doi:10.25270/con.2022.01.00003

Received September 2, 2021. Accepted October 1, 2021.

\section{DISCLOSURES:}

The authors report no relevant financial relationships.

\section{CORRESPONDENCE:}

Judith Volcy, DO Morehouse School of Medicine, 720 Westview Drive, SW Atlanta, GA 30310 (jvolcy@ msm.edu)

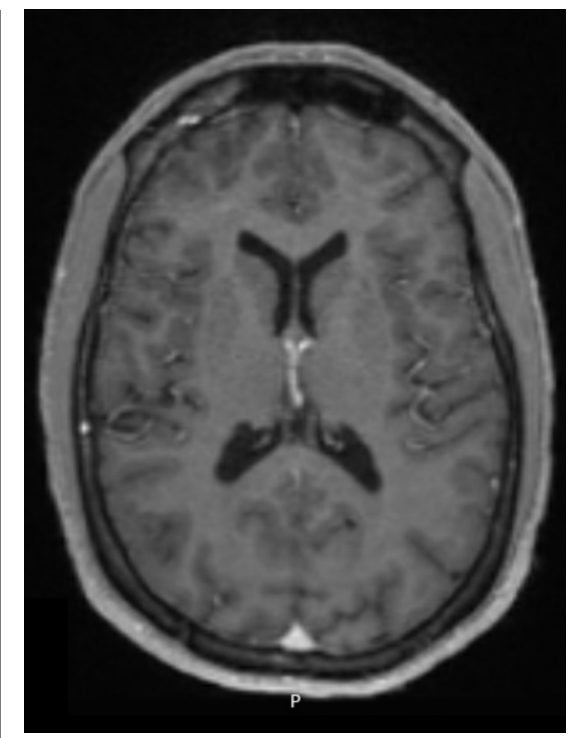

Figure. The patient's MRI scan showed advanced basal ganglia mineralization for his age.

haloperidol, $5 \mathrm{mg}$, lorazepam, $1 \mathrm{mg}$, and diphenhydramine, $50 \mathrm{mg}$. He was then discharged home. The patient followed up with his primary care provider, where he reported continued cannabis use.

\section{Discussion}

Cannabis is the most widely used illicit psychoactive agent in the world. In the United States, there are varying levels of legality across the states, and usage has become more widespread. It is well known that there is a significant association between cannabis use and psychoactive disorders. The findings of our case report highlight the importance of increased awareness of the adverse effects of cannabis use, particularly the induction of psychosis.

About $20 \%$ of teenagers and young adults worldwide have reported cannabis 
use at least once per week or heavy use (ie, > 100 occasions). ${ }^{2}$ Moore and colleagues reviewed 35 studies worldwide and found a $40 \%$ risk of psychosis in participants who had ever used cannabis. ${ }^{2}$ Another study conducted by Starzer and colleagues showed that $32 \%$ of participants with cannabis-induced psychosis were later diagnosed with bipolar/schizophrenia. ${ }^{3}$ Participants using cannabis had the highest conversion to bipolar/ schizophrenia (47.4\%) than participants using any other substance. ${ }^{3}$ Participants aged 16 to 25 years and men were at the highest risk of any substance-induced psychosis, while individuals aged older than 51 years were at the lowest risk. ${ }^{3}$

\section{Conclusions}

Our case supports other data being published in the United States. Frequent cannabis users are at the greatest risk of developing psychosis. Subtle cognitive deficits can be noted at least 7 days after heavy cannabis use; after 28 days, virtually no deficits can be noted, suggesting reversible neurologic effects. ${ }^{4}$

Synthetic cannabis is much more potent than natural cannabis and does not include the cannabidiol component that otherwise counteracts the psychoactive components of the tetrahydrocannabinol. ${ }^{5}$ Cannabis-induced psychosis should be considered in the differential diagnosis of patients who present with encephalopa- thy, and patients should be counseled on potential adverse effects of cannabis use.

\section{References}

1. World Drug Report 2018. UN Office on Drugs and Crime. June 2018. Accessed January 7, 2022. https://www.unodc.org/ wdr2018/prelaunch/WDR18_Booklet_1_EXSUM.pdf

2. Moore TH, Zammit S, Lingford-Hughes A, et al. Cannabis use and risk of psychotic or affective mental health outcomes: a systematic review. Lancet. 2007;370(9584):319328. https://doi.org/10.1016/s01406736(07)61162-3

3. Starzer MSK, Nordentoft M, Hjorthøj C. Rates and predictors of conversion to schizophrenia or bipolar disorder following substance-induced psychosis. Am J Psychiatry. 2018;175(4):343-350. https://doi. org/10.1176/appi.ajp.2017.17020223

4. Nader DA, Sanchez ZM. Effects of regular cannabis use on neurocognition, brain structure, and function: a systematic review of findings in adults. Am J Drug Alcohol Abuse. 2018;44(1):4-18. https://doi.org/10.10 80/00952990.2017.1306746

5. Altintas $M$, Inanc $L$, Oruc GA, Arpacioglu S, Gulec $\mathrm{H}$. Clinical characteristics of synthetic cannabinoid-induced psychosis in relation to schizophrenia: a single-center cross-sectional analysis of concurrently hospitalized patients. Neuropsychiatr Dis Treat. 2016;12:1893-1900. https://doi.org/10.2147/ ndt.s107622 